\title{
JURADOS CIUDADANOS Y ORGANISMOS GENÉTICAMENTE MODIFICADOS
}

\author{
EMILIO LUQUE \\ Universidad Nacional de Educación a Distancia
}

\author{
PALABRAS CLAVE ADICIONALES \\ ADDITIONAL KEYWORDS \\ Democracia deliberativa, OGM, Jurados Deliberative Democracy, GMO, Citizens \\ ciudadanos, Conocimiento experto. \\ Juries, Expertise and Citizenship.
}

RESUMEN. Una sociología política de la alimentación debe analizar los procesos a través de los cuales se alcanzan las decisiones de regulación y control alimentario y examinar el potencial de sus alternativas. Se describen aquí cinco experimentos democráticos en los que se ha empleado uno de los dispositivos deliberativos más prometedores en condiciones de alta complejidad cognitiva, los llamados jurados ciudadanos, con la participación de campesinos hindúes y brasileños y consumidores británicos, y centrados en los Organismos Genéticamente Modificados. Estos procesos, caracterizados por el acceso de los miembros del jurado a testigos expertos presentados por los distintos participantes en la controversia, muestran la enorme capacidad de los ciudadanos "de a pie" para articular el análisis de un problema complejo, reevaluar sus riesgos y recontextualizar el uso de los OGM. En todo caso, la democracia deliberativa no es una solución sencilla a la crisis política y epistémica que subyace a la crisis alimentaria, sino que marca un cambio de paradigma hacia una democracia experimental, en la que las instancias de representación de lo público (y los públicos) se multiplican.

\begin{abstract}
A political sociology of food must look into the processes whereby regulatory decisions on food are made, and also into the democratic potential of their alternatives. Five "experiments in democracy" are described, in which a promising deliberative device has been used: citizens' juries. Indian and Brazilian peasants, on the one hand, and British consumers in the other took part in them, and they focused on Genetically Modified Organisms. These processes, whose defining trait is granting jurors access to expert witnesses presented by all stakeholders in the controversy, show the impressive ability of ordinary citizens to articulate their analysis of complex issues, a reassessment of risks, and a recontextualization of the use of GMOs. At any rate, deliberative democracy is not a magic bullet for the epistemic and political crisis that underlies food crisis; instead, it points at a paradigm change towards an experimental democratic polity in which the instances of representation of the public and publics are multiplied.
\end{abstract}

E-mail: eluque@poli.uned.es

Revista Internacional de Sociología (RIS)

Tercera Época, Nº 40, Enero-Abril, 2005, pp. 231-246. 
R I S

REVISTA INTERNACIONAL DE SOCIOLOGIA

NN 40, ENERO-ABRIL, 2005

EMILIO LUOUE

\section{INTRODUCCIÓN}

Pretender que el ciudadano sea capaz de entender y dirigir el curso de los acontecimientos es como querer que un hombre obeso sea bailarín, decía Walter Lippmann (1925). «El hombre individual no tiene opinión sobre los asuntos públicos [y] no hay la menor razón para suponer, como han pensado los demócratas místicos, que la suma de las ignorancias individuales en masas de gente pueda producir una fuerza directora estable en los asuntos públicos», señalaba en The Phantom Public. La complejidad de las decisiones asociadas a la producción y el consumo de alimentos, que se ha expuesto en gran parte de las contribuciones a este volumen (véasen especialmente los artículos de Jesús Contreras, Mábel Gracia y Cecilia Díaz al respecto), parecen hacer aún más firme este juicio. ¿Cómo confiar a los ciudadanos las decisiones sobre la regulación de los herbicidas, o de la trazabilidad de las carnes procedentes del ganado bovino, o de los Organismos Genéticamente Modificados (OGM), si no son en absoluto expertos en bioquímica, en priones o en ácido desoxirribonucleico?

Sin embargo, la forma concreta de democracia hacia la que Lippmann dirigía sus andanadas, el voto por mayoría sin ningún proceso de información previo (salvo, quizá, el de las campañas electorales y los medios de masas, que pueden muy bien ser ejemplos de desinformación), no es necesariamente el único dispositivo posible para el debate público y la toma de decisiones. Si imaginamos otros procesos alternativos por los que los ciudadanos pudieran alcanzar decisiones bien informadas, la posibilidad de poner en sus manos el gobierno de sus asuntos comunes nos parecería seguramente más razonable. Los procedimientos que organizan en la práctica los asuntos públicos son decisivos, como señala Michael Saward (2003), por mucho que hayan .quedado a menudo fuera de la reflexión política, normalmente centrada en debates en torno a principios, y no a su puesta en práctica. Sin embargo, «los principios democráticos se hacen vivos (son "vividos") a través de la mediación de mecanismos o dispositivos formales de decisión que están diseñados para activarlos y que se justifican en esos términos. La percepción de su utilidad como principios dependerá en gran medida del funcionamiento de dichos dispositivos» (Saward, 2003: 166). El quién, cómo y dónde concreto de las decisiones públicas es el marco de sentido mismo de las democracias, el espacio en que se construye su legitimidad y su eficacia.

Por otro lado, la perspectiva tecnocrática, que considera necesario trasladar a los expertos las decisiones sobre aquellos campos cuya complejidad supera las capacidades de ciudadano medio, es quizá especialmente difícil de aceptar en los terrenos más cercanos, más íntimos, más cotidianos. Determinados procedimientos médicos en casos terminales son ejemplos extremos de esta gestión externa del propio cuerpo, un espacio sobre el que a menudo uno mismo tiene, paradójicamente, muy poco control. En este sentido, el impulso de recuperación de formas de control de los ciudadanos sobre su entorno cercano supone un primer nivel de cuestionamiento de la regulación de la producción y del consumo de alimentos. 
Pero también, como han puesto asimismo de manifiesto varias de las contribuciones a este número, el sistema que articula la producción y el consumo de alimentos constituye un nodo crucial de los procesos de globalización, sus asimetrías y sus consecuencias perversas ${ }^{1}$. Movimientos como el de la slow food, o el rechazo organizado a la comida basura, ${ }^{2}$ o a los organismos modificados genéticamente, deben entenderse, así, desde las claves políticas de la resistencia a esta red hegemónica de factory farming, obesidad y hambre, subsidios al agronegocio y aranceles a los productores del Tercer Mundo, entre otros hilos.

En la intersección de estos dos ejes, los procedimientos por los que los ciudadanos acceden a los problemas asociados al sistema alimentario y a las decisiones sobre ellos se tornan cruciales. La representación reflexiva e informada de la naturaleza de los asuntos públicos queda en principio fuera de los mecanismos concretos del voto por mayoría. Se deja (de manera más bien implícita) a los propios ciudadanos la tarea de informarse sobre los asuntos acerca de los cuales deberán decidir después, normalmente en el sentido de seleccionar representantes de los que esperan que tomen las decisiones adecuadas. La «esfera pública» - que en la definición algo tautológica de Habermas (1994) es la «esfera de los individuos privados que se organizan como público»—no está institucionalizada en un sentido positivo ${ }^{3}$. Las iniciativas públicas, en forma por ejemplo de campañas educativas, son minúsculas si las comparamos con los enormes medios movilizados por las grandes corporaciones; en todo caso, responden a un mandato difuso de ilustración ciudadana que tiene algún sentido en tradiciones y culturas políticas como la escandinava ${ }^{4}$, pero que en nuestras mediterráneas latitudes es notablemente anémica.

Podemos identificar al menos tres factores que plantean la necesidad de renovar las formas concretas en las que se desarrolla el juego democrático. Por un lado, la creciente complejidad de los asuntos a los que nos enfrentamos, que

\footnotetext{
${ }^{1}$ Un caso que condensa de manera casi perfecta esta perspectiva es el del maíz estadounidense. Por un lado, las cuantiosas subvenciones a su producción hacen que penetre de manera destructiva en amplias regiones rurales del Tercer Mundo, contribuyendo a los desplazamientos masivos hacia las zonas urbanas, cada vez más insostenibles. Por otro, la disponibilidad de subproductos como el sirope de maíz con alto contenido en fructosa es probablemente decisivo en la pandemia de obesidad y diabetes tipo II (Fields, 2004; Bray et al., 2004).

${ }^{2}$ Cómo no recordar en este punto a José Bové y su fotogénica combinación de pipa y bigote, tan antipática para McDonald's.

${ }^{3}$ Aunque sí en el sentido de proteger, bajo la forma de la libertad de expresión, esa «conversación anónima» que la constituye, de acuerdo con Seyla Benhabib (1996).

${ }^{4}$ Los llamados círculos de estudio y los altos niveles de lectura de periódicos, ambos ampliamente subvencionados desde las agencias estatales, son excelentes ejemplos de esta tradición de «ilustración pública»; de acuerdo con Milner (2002), el nivel de información y prácticas cívicas que promueven explicaría «cómo funciona la democracia», frente al conocido análisis de Robert Putnam (2002) en términos de corales y ligas de bolos.
} 
Bruno Latour condensa en la expresión «el mundo como experimento colectivo» (Latour, 2001), un experimento que ha desbordado hace mucho tiempo los confines del laboratorio y tiene lugar "a escala 1:1", en el lenguaje de los topógrafos. Piénsese en una de las transformaciones más relevantes para el medio ambiente, la proliferación de especies invasoras: ningún ecosistema in vitro puede predecir las devastadoras consecuencias de introducir un tipo determinado de alga tropical ${ }^{5}$ en el Mediterráneo, o las aparentemente inofensivas chumberas en Australia. Esta complejidad no puede ser fácilmente clausurada mediante una acumulación mayor de conocimiento experto, puesto que los mismos procesos de producción y legitimación de ese conocimiento son precisamente parte integral del juego político (véanse, entre otros muchos, los trabajos de Sheila Jasanoff al respecto).

Por otro lado, es obligado replantearse la calidad democrática de la esfera pública en tiempos de oligopolio de los medios de comunicación de masas, en sincronía con una estrategia de simplificación en los mensajes de partidos y medios, y con la concentración del poder empresarial en enormes conglomerados transnacionales. La «gestión científica de la política por especialistas en "declaraciones de telediario" (sound bites)» (Ackerman y Fishkin, 2002: 13) es parte central de esta baja calidad del lenguaje público de las democracias.

Por último, como destaca Fung (2003), el tipo de innovaciones democráticas, multiformes, experimentales y localizadas que aquí nos ocupan ofrecen una alternativa más operativa y susceptible de ser puesta en práctica en forma de iniciativas «realmente existentes», frente a las grandes críticas (Fung las denomina «tectónicas») de las democracias actuales como la revitalización de la sociedad civil argumentada en el macrodiagnóstico de «déficit de capital social» de Robert Putnam (2002), entre otros.

Entre estos procedimientos ${ }^{7}$ innovadores a la hora de organizar el debate público y la toma de decisiones de forma deliberativa estarían las llamadas "encuestas deliberativas»" (Luskin et al., 2002), las «conferencias de consenso» (Joly et al., 2003) y los jurados ciudadanos. En particular, estos últimos muestran un gran potencial en lo que Saward (2003) denomina un estadio o fase deliberativa,

\footnotetext{
${ }^{5}$ La conocida y letal Caulerpa taxifolia. Una excelente introducción al problema de las especies invasoras, la segunda causa de pérdida de biodiversidad tras la desaparición del hábitat, puede hallarse en Baskin (2002).

${ }^{6}$ El día en el que terminó la redacción de este artículo, el magnate de los media Rupert Murdoch trataba de adquirir la parte que no controlaba de la cadena FOX (parece que central en la estructuración del debate público en Estados Unidos por parte de los conservadores) por la módica cantidad de 6,000 millones de dólares.

${ }^{7}$ El lector encontrará en Colino y del Pino (2003) una buena introducción comparada al despliegue de estos métodos participativos en el ámbito local europeo.

${ }^{8}$ Deliberative polling, que como anuncia la página web dedicada a este tema en la Universidad de Texas en la que trabaja Fishkin, es «marca registrada» por este último, nada menos.
} 
no necesariamente ligada a la toma de decisiones (que puede estar instrumentada mediante los procedimientos más «clásicos» del voto y la selección de representantes), pero que podría enriquecer substancialmente el debate público, y proporcionar un acercamiento a esa perspectiva ideal (que es siempre una especie de ficción democrática, útil quizá, pero cuya nostalgia conduce a la melancolía política) que tendrían ciudadanos bien informados, atentos e interesados por el bien común (y con bastante tiempo libre ${ }^{9}$ ). En esencia, los jurados ciudadanos ofrecen a un grupo, usualmente de entre diez a quince personas, la posibilidad ${ }^{10}$ de recibir el testimonio de testigos expertos, designados por los interesados en el asunto tratado (los ya ubicuos stakeholders), cuestionar a dichos testigos, reflexionar colectivamente sobre el problema, y emitir un "dictamen", que no es un veredicto de culpabilidad o inocencia sino un conjunto de diagnósticos y propuestas.

Hay un elemento más que explica el interés aquí reseñado por los jurados ciudadanos, especialmente en el terreno de una sociología política de la alimentación, y de la producción de conocimiento sociológico en general. Los jurados ciudadanos, en este sentido, se enmarcan en los procesos de investigación-acción que rompen con la lógica cientifista que contribuye a separar objeto sociológico de acción ciudadana, asignándoles un estatuto pasivo, y filtrando los modos de conocimiento no académico-técnicos (Wakeford, 2002). En particular, en relación con la "ciudadanía ecológica" (Luque, 2005, en prensa), las metodologías cualitativas y cuantitativas al uso muestran serios límites para recuperar las prácticas comunicativas que conforman la esencia misma de la ciudadanía. Pero para situar adecuadamente el potencial de los jurados ciudadanos, lo mejor es que describamos con detenimiento su aplicación al tema de este volumen en cinco casos concretos, lo que nos ocupará en el siguiente epígrafe.

\section{EXPERIENCIAS DE JURADOS CIUDADANOS Y OGM}

El agrio debate en torno a los organismos genéticamente modificados reúne en su grado más intenso gran parte de las características de las crisis alimentarias actuales, como exploran en este volumen Merdji y Debucquet, Espeitx y Herrera.

\footnotetext{
${ }^{9}$ Recuérdese la queja de Oscar Wilde: el socialismo cuesta demasiadas tardes libres.

${ }^{10}$ No entraré en exponer y valorar las muchas críticas y contrapropuestas en torno a este conocido asunto de la democracia representativa. Mi intención es sólo explorar cómo dispositivos de representación del público distintos de esa mezcla de elitismo y democracia del gobierno representativo (Manin, 1997) modifican la perspectiva sobre los problemas alimentarios, y no la de proponer su extensión inmediata a todas las áreas de las decisiones democráticas. Sólo señalaré aquí que sus defensores son conscientes, entre otras muchas cosas, de los costes que un jurado ciudadano inclusivo y viable supone en la práctica, que incluyen "pago de honorarios, servicios de guardería, o edificios con accesos para minusválidos" (Wakeford, 2002).
} 
Para no reiterar los argumentos allí apuntados, lo que resulta más interesante para los fines de este artículo es 1) la "barrera epistémica" que presentaría la esotérica matriz de ciencia y tecnología en la que nacen los OGMs para su comprensión por parte de los ciudadanos legos, 2) el nudo gordiano que suponen en la madeja de desarrollo del Tercer Mundo, hegemonía del agronegocio, sostenibilidad y riesgo alimentario.

Se describen a continuación cinco procesos de jurados ciudadanos que tratan de ilustrar perspectivas muy distintas sobre los alimentos provenientes de OGM. Por un lado, en tres de ellos están implicados los potenciales productores de alimentos derivados de transgénicos, frente a otros dos de potenciales consumidores. En los primeros fueron llamados a participar ciudadanos del Tercer Mundo, mientras que los segundos se desarrollaron en Inglaterra. Y, sin embargo, como el lector podrá apreciar, algunas de las pautas del proceso y el veredicto de los jurados muestra "aires de familia" de notable interés, sobre los que regresaremos más abajo. Anotaremos con algo de detalle los procesos concretos de su puesta en práctica, porque, como hemos venido señalando, aquí también the devil is in the details.

\section{El GM Jury}

Una de las experiencias más relevantes para esta breve contribución a una sociología política de la alimentación es la que constituye el doble jurado ciudadano organizado durante el verano del año 2003 en dos localidades inglesas de los distritos de Hertfordshire y Tyneside. El proceso fue patrocinado por ONGs (Greenpeace y la Consumers' Association -Asociación de Consumidores-), grandes empresas (Unilever) y el grupo de cooperativas Co-op Group. La intención expresa de los organizadores era «proporcionar un input público deliberativo al debate público sobre los OGM, patrocinando de forma conjunta un jurado ciudadano capaz de marcar la agenda pública ${ }^{11}$ sobre los alimentos y cultivos genéticamente modificados» (PEALS, 2003).

Antes de que comenzaran las sesiones del jurado se desarrolló un proceso preparatorio en tres fases: la creación de un comité supervisor (Oversight Panel), la selección de los miembros del jurado, y la de los testigos. El Comité estuvo encargado de asegurar la calidad democrática en todos los momentos del proceso, y se reveló como mediador clave en la construcción de confianza que hace relevante este proceso para el público más amplio. Participaban en él representantes de la industria biotecnológica como Syngenta, académicos y asesores políticos. La selección del jurado se realizó a partir de un listado de 5.800 personas, aleatoriamente escogidas a partir del censo electoral, a las que se envió una carta de

\footnotetext{
${ }^{11}$ El conocido agenda-setting de los politólogos.
} 
invitación en la que se describían las fechas y el proceso del jurado ciudadano, pero que no mencionaba el asunto del que se ocuparía. Los organizadores ${ }^{12}$ del instituto PEALS de la Universidad de Newcastle agruparon las respuestas por categorías ${ }^{13}$ de género y edad, y seleccionaron, aleatoriamente de nuevo, los integrantes del jurado. En paralelo, el jurado preparó una lista equilibrada de expertos, con el doble criterio de que fueran capaces de exponer con claridad la evidencia que presentaban, $y$ de interactuar con el jurado, que tendría la posibilidad de interrogarles en profundidad. Entre estos testigos se encontraban científicos, representantes de agricultores, ONGs, consultores y responsables de la industria biotecnológica.

Las sesiones del jurado tuvieron lugar durante diez tardes, con una duración de dos horas y media, cada una en el horario más compatible posible con las distintas situaciones laborales y vitales. En estas sesiones, la figura del "facilitador" es relevante, en la medida en que hacía más inclusivas, fructíferas y participativas las discusiones y reuniones, con especial hincapié en posibilitar que los miembros del jurado menos dados a hacerse oír pudieran contribuir al proceso. Para evitar que los jurados se perdieran en la jerga técnica de alguno de los testimonios, uno de los participantes le enseñaba una "tarjeta amarilla", indicándole con ello que debía explicar de forma más accesible sus argumentos.

Finalmente, durante el arduo proceso de redactar un informe conjunto, con discrepancias incluidas, se les pidió que clasificaran por orden de importancia las recomendaciones que acordaban en pequeñas "comisiones delegadas" de cuatro o cinco personas. Estas recomendaciones eran posteriormente sometidas a una votación anónima. Entre las conclusiones de los jurados desarrollados en Tyneside y Hertfordshire de forma independiente, aparecían similitudes notables, que resumimos a continuación:

a) Por un lado, la cuestión de los OGM se vinculaba a un sistema alimentario con rasgos preocupantes, de los que se destacaba la sobreutilización de antibióticos en la ganadería y la desaparición de los pequeños y medianos agricultores, o su dependencia creciente de las transnacionales de la biotecnología alimentaria.

b) La incertidumbre respecto del impacto medioambiental de la producción y consumo a gran escala de los OGM hizo que ambos jurados optasen por aplicar el "principio de precaución", de manera totalmente explícita en el caso de Hertfordshire. Ambos jurados consideraban que los ensayos y controles debían ser

\footnotetext{
${ }^{12} \mathrm{El}$ autor quiere agradecer aquí a Tom Wakeford y a Fiona Hale, dos de los más activos investigadores en el terreno de los jurados ciudadanos, la "democracia tecnológica" y las formas participativas de desarrollo endógeno, su amable disposición a lo largo de la preparación de este artículo.

${ }^{13}$ Con números entre los diez y los quince integrantes, los jurados ciudadanos no pueden pretender una representatividad estadística del cuerpo social y político, sino que estarían en la lógica de "representación cognitiva" de lo que Robert Dahl denominó minipopulus, asambleas de en torno a mil ciudadanos que debatirían durante un año sobre un tema concreto (Dahl, 1991: 408-9).
} 
mucho más exhaustivos y de mayor duración antes de autorizar la importación y el uso indiscriminado de OGMs.

c) La financiación de la investigación por parte de la industria biotecnológica hacía que aquélla no fuera fiable, lo que debía ser contrapesado por una acción pública de producción de conocimiento científico.

d) Los argumentos de las multinacionales en el sentido de que los OGMs contribuirían a mejorar la situación de los campesinos en países del Tercer Mundo fueron rechazados, resituando el problema en una distribución desigual de los recursos.

e) El desplazamiento del riesgo hacia agricultores, por un lado, y consumidores por otro, fue también rechazado, proponiendo medidas de responsabilización de empresas como Monsanto de los problemas que pudieran acarrear el uso de sus semillas y otros "productos".

\section{Karnataka: flora edáfica microbiana y campesinas analfabetas}

Organizado por ActionAid India, este jurado ciudadano se llevó a cabo en una aldea del Estado hindú de Karnataka (Pimbert et al, 2001). En esta ocasión, el jurado estuvo conformado por catorce pequeños agricultores y agricultores marginales (principales afectados por los OGM), seis hombres y ocho mujeres, procedentes de distintos grupos socioeconómicos y tradiciones agrícolas. Entre los testigos expertos se encontraban miembros de institutos científicos, empresas de biotecnología (Monsanto), ONGDs, sindicatos agrícolas y agencias gubernamentales. Tras cuatro días de testimonios e interrogatorios, supervisados por un panel, el jurado emitió su veredicto, que respondía a la pregunta ¿sembrarian en sus campos las nuevas semillas comerciales (genéticamente modificadas) propuestas por el Departamento Indio de Biotecnología y la empresa Monsanto? El voto secreto arrojó un resultado de 4 síes y 9 noes, más un voto nulo.

Pero, como corresponde a un jurado ciudadano, no se trataba sólo de dar una respuesta negativa o positiva, sino de señalar vías posibles de aceptación para estas "nuevas semillas". Las principales recomendaciones en este sentido incluían el que no se dañara a los microbios e insectos benéficos, ni el medio ambiente en general; la realización de ensayos de campo durante períodos de cinco a diez años; el que las "nuevas semillas" no afectaran a cultivos vecinos o a los que se realizaran en el mismo terreno posteriormente. Un elemento importante es la inclusión de los agricultores en la definición, realización y evaluación de los ensayos de campo. Las posturas de los jurados iban desde los que rechazaban de pleno las semillas genéticamente modificadas hasta los que aceptarían su cultivo, siempre que las empresas les aseguraran contra los riesgos asociados (con compromisos escritos y respaldados económicamente, por ejemplo, comprando las cosechas al doble de su precio en caso de que hubiera problemas). 
La posición del jurado respecto de las compañías multinacionales y la biotecnología, y la posibilidad de reconstruir vínculos de confianza hacia ellas, iba desde una reticente y minoritaria disposición a aceptar colaborar con las empresas hasta el mayoritario rechazo frontal, asociado entre otras cosas a la actuación de las transnacionales del agribusiness en la Organización Mundial del Comercio y al debate sobre las patentes y la propiedad intelectual. De acuerdo con estos participantes, las compañías multinacionales terminarían por eliminar toda capacidad de decisión autónoma de los agricultores.

Este jurado en particular supuso para el autor una confirmación decisiva de las capacidades latentes en la ciudadanía para representar adecuadamente sus problemas, por complejos que estos sean. Las campesinas que participaban en él, como Surmangala (que aparecía fotografiada en el informe), habían incluido con toda naturalidad entre sus consideraciones el que debía asegurarse que los OGM no dañaran la flora edáfica (esto es, presente en el suelo) microbiana, y habían interrogado a los biotecnólogos sobre la resistencia diferencial de los insectos expuestos a mayores dosis de pesticidas basados en glifosatos (a los que la soja de Monsanto sería resistente). Estos campesinos hindúes analfabetos parecían aprender a bailar (por usar las palabras de Lippmann) más rápido y con mayor elegancia de lo que se podría esperar desde las perspectivas más desencantadas del análisis político. No se trata, sin embargo, de que los participantes pasen a ser expertos en genética o botánica, sino de que tengan la oportunidad de reconstruir el problema en torno a las categorías relevantes para su experiencia y horizonte vital cotidianos.

\section{Prajateerpu: los escenarios del desarrollo}

Prajateerpu quiere decir "veredicto del pueblo" en uno de los idiomas del Estado hindú de Andra $\left(\operatorname{Pradesh}^{14}\right)$. Nos encontramos esta vez ante un ejercicio democrático más holístico y a más largo plazo, puesto que incluía un modelo de selección entre "escenarios" o "visiones de futuro" para el desarrollo de Andra (Pimbert y Wakeford, 2002). Cada uno de los tres escenarios era propuesto por determinados stakeholders. Así, la "Visión 2020" del gobierno de Andra Pradesh, respaldado por un préstamo del Banco Mundial, se centraba en la reducción de la población agrícola de un setenta a un cuarenta por ciento, la mecanización e introducción de OGMs, y la concentración de las explotaciones. La propuesta de IFOAM (la Federación Internacional de Movimientos por la Agricultura Orgánica), con el apoyo del Centro Internacional del Comercio se basaba en una agricultura sostenible, pero vinculada a la demanda del Norte de productos orgánicos. La última de las propuestas, en la que resuenan las ideas de Gandhi y que se presentaba desde sindicatos campesinos y ONGDs de corte más crítico, destacaba la producción

\footnotetext{
${ }^{14}$ Pradesh significa "Estado".
} 
local de alimentos, una agricultura de bajos insumos, y la autosuficiencia de las comunidades rurales afectadas. El método de presentación de estas alternativas fueron unos vídeos elaborados por el Instituto Internacional para el Medio Ambiente y el Desarrollo (IIED ${ }^{15}$ son sus siglas en inglés) bajo la supervisión de los proponentes que formaban parte de un consejo o comité, como en general lo hicieron con todo el proceso, presidido por un juez - antiguo presidente de la Corte Suprema de la India-jubilado. La fuerte estratificación social de la India llevó en esta ocasión a asegurar una cierta representatividad del Andra rural, incluyendo a miembros Dalit (de la casta de los intocables, el escalón inferior de un sistema supuestamente abolido) e indígenas (conocidos como adivasi), así como haciendo que dos tercios de los miembros fueran mujeres, las cuales tienen el papel central en el trabajo agrícola.

Durante cuatro días, el jurado escuchó e interrogó a doce testigos, desde altos funcionarios de Andra, hasta representantes de IFOAM, pasando por biotecnólogos de Syngenta. Las conclusiones del jurado se encaminaron en la dirección de aumentar la autosuficiencia y el control comunitario sobre los recursos, poner en valor el conocimiento local, las instituciones y las habilidades autóctonas, y mantener la diversidad y la salud ${ }^{16}$ de la tierra. Rechazaron explícitamente los desplazamientos masivos de campesinos, la introducción de cultivos genéticamente modificados, incluyendo el "arroz dorado" (más rico en precursores de la vitamina A) y el algodón $\mathrm{Bt}$ (supuestamente más resistente a las plagas de insectos).

Es destacable en este caso el papel de los facilitadores locales, hablantes de la lengua autóctona (Telegu), y que habían recibido una cuidadosa formación en las múltiples habilidades necesarias para llevar a buen término el proceso; en la utilización de tecnologías audiovisuales para la presentación de los escenarios y parte de los testimonios expertos; también la localización del proceso en un entorno conocido (una granja de formación agrícola); pero, sobre todo, la subversión de las jerarquías implícitas y explícitas activas en el proceso político.

\section{Belem do Pará}

En septiembre de 2001 tuvo lugar este jurado ciudadano en la capital del Estado amazónico de Pará, entre cuyos patrocinadores se encontraban el conocido "movimiento de los sin tierra", centrales sindicales y ONGDs como ActionAid

\footnotetext{
${ }^{15}$ Las otras entidades encargadas de la organización del proceso fueron la Universidad de Hyderabad, la Coalición en Defensa de la Diversidad, el Plan Estratégico Nacional para la Biodiversidad, entre los hindúes, y el Instituto para los Estudios del Desarrollo (IDS) británico.

${ }^{16}$ La cosmovisión de "salud" espiritual aplicada a la tierra es mucho más rica de lo que los modelos de conocimiento occidentales permiten. Piénsese en las chacras (terrenos de cultivo) peruanas, que según aseguran los quechuas deben estar "calientes" para ser productivas, gracias, entre otras cosas, a los juegos de los niños.
} 
Brasil. La selección de los miembros del jurado se realizó en esta ocasión a partir de los miembros de seis asociaciones comunitarias, de las que se seleccionaron aleatoriamente cuatro personas. De esta lista de veinticuatro, se sortearon los siete participantes, cuatro mujeres y tres hombres. El "juez", papel que desempeñaba el decano de la Facultad de Derecho de la Universidad Federal de Pará, planteó el "caso" ante el jurado, que incluía una definición de los OGM, sus usos, y las cuestiones a las que el jurado debía responder, que merece la pena transcribir aquí:

- “¿Pueden resolver los OGM el problema del hambre?

- ¿Pueden mejorar la seguridad alimentaria de los pequeños agricultores?

- ¿Existe evidencia suficiente de que los OGM no amenazan el medio ambiente?

- ¿Es lo bastante transparente y cauto el proceso de liberalización y uso comercial de los OGM?"

En el caso de este jurado, el formato se aproximó en la práctica, y no sólo conceptualmente, al de un jurado clásico. Así, hubo "defensa" (un biotecnólogo de la Universidad Federal de Pará) y un "fiscal" (un abogado del Ayuntamiento de Belem). Tras sus alegatos, presentaron a tres testigos cada uno, que fueron después interrogados por todos los participantes. Dos biotecnólogos y un miembro de la comisión nacional de bioseguridad lo hicieron por la defensa, y un economista, un genetista y un antropólogo por parte de la acusación. Finalmente, el jurado votó cada una de las cuestiones planteadas: la respuesta unánime fue negativa.

\section{Algunos rasgos comunes}

Una lectura detenida de las decisiones finales de los jurados esquemáticamente resumidos aquí, junto con otras experiencias relacionadas (Pimbert y Wakeford, 2002; Joly et al., 2003) muestra algunas pautas interesantes. En este punto destacaré seis de estos rasgos, que se pueden sintetizar como sigue: la reevaluación del riesgo en términos de los actores implicados, el desplazamiento de la escala temporal de referencia, la recontextualización del uso de los OGM, la constatación del potencial de análisis reflexivo por parte de ciudadanos presuntamente sin recursos (incluyendo los cognitivos) cuando el diseño institucional incorpora flujos de información de alta calidad, la puesta en valor del conocimiento y las instituciones locales, y la capacidad de reorientación de los diagnósticos y prescripciones que se deriva de este análisis.

Quizá la mejor manera de acercar al lector al modo en que estas pautas emergían en los debates con los expertos y las reflexiones internas del jurado sea transcribir por extenso esta reflexión de Deevenamma, una de las campesinas que formaron parte de Prajateerpu: 


\title{
R I S
}

\begin{abstract}
"Hace mucho tiempo, cuando hacíamos nuestra propia agricultura con nuestros propios métodos, producíamos también suficiente y comíamos y teníamos una vida cómoda con ropa y comida y todo. Entonces pasamos a la agricultura química porque nos prometieron que daría buenos rendimientos y más producción. Así que pasamos a este tipo de agricultura, pero poco a poco tenemos que incrementar nuestros insumos en forma de comprar cada vez más fertilizantes y pesticidas y muchas otras formas de gestión.

Ya tenemos dolor en las articulaciones y otros problemas de salud por esta agricultura química. Ahora nos decís que tenemos otro tipo más de agricultura: los cultivos GM. También nos decís ahora que habéis creado nuevas variedades. No sabemos si son seguras para nuestro consumo. A causa de la agricultura química nuestro propio pasto ya no es comestible para nuestro propio ganado por los residuos de los pesticidas. A nuestro ganado no le gusta el forraje de alto rendimiento. Si les dáis OGM, que matan a un insecto cuando come una hoja, ¿cómo podéis estar seguros de que no envenenará o matará a nuestro ganado o a nosotros cuando los usemos para el consumo humano o como alimento para el ganado? Todas estas son dudas genuinas que tenemos. Estoy segura de que nuestros propios métodos agrícolas son más seguros que estos nuevos que no han sido probados".
\end{abstract}

Desde una perspectiva de conjunto, quizá lo que destaca en todos los ejercicios es el potencial de "explosión democrática" que late en todos los casos considerados. El síntoma de esta capacidad expansiva del ejercicio de los jurados ciudadanos es la de ir más allá de las preguntas formuladas en el lenguaje de los "sospechosos habituales" de la política representativa, cambiando las coordenadas del problema desde los parámetros habituales de crecimiento, conocimiento experto, productividad, coste-beneficio o control hacia los de precaución, seguridad, ciudadanía, salud y equidad.

\section{LA DEMOCRACIA DELIBERATIVA COMO (DI)SOLUCIÓN DE LA(S) CRISIS ALIMENTARIA(S)}

¿Se trata, pues, simplemente de multiplicar las instancias deliberativas, jurados ciudadanos, presupuestos participativos, y otros dispositivos aún por inventar? ¿Resolvería esto, en el marco del tema central de este volumen, la crisis de la seguridad alimentaria o las pandemias nutricionales, o el déficit democrático al que están asociadas?

En su fascinante libro sobre las "metáforas de la vida cotidiana", Lakoff y Johnson (1980) relatan cómo un estudiante no estadounidense había entendido la expresión "la solución de tus problemas" (the solution of your problems) a partir del concepto químico de "solución", es decir, la "acción y efecto de disolver" una substancia en un líquido. Desde esta proyección metafórica, no se espera de los 
problemas que queden cerrados para siempre, sino que estén siempre presentes en concentraciones variables, a veces imperceptibles, precipitando otras, haciendo inevitable la tarea de periódicas agitaciones. Si uno entiende los problemas desde esta "metáfora química", "en lugar de encaminar las energías hacia la resolución definitiva de los problemas, se trataría de encontrar los catalizadores que permitan disolver los más acuciantes durante el mayor período de tiempo posible, sin precipitar otros peores" (Lakoff y Johnson, 1980: 143-4).

Pues bien, la aplicación de dispositivos democráticos de alta calidad cognitiva como los jurados ciudadanos a terrenos como el alimentario debe entenderse como una "solución" de este tipo. Los jurados permiten la puesta en cuestión, por parte de ciudadanos bien informados, de las claves temporales, de las asignaciones de riesgos, de los parámetros "técnicos" con los que se evalúan los problemas públicos desde las redes tecnocráticas de expertos; pero no pueden llevar a decisiones sistemáticamente "mejores", es decir, más legítimas, eficientes y seguras, si no forman parte de una constante convocatoria de asambleas, de dispositivos de representación (en su doble sentido político y epistemológico) que conforman la visión política de autores como Bruno Latour (1999) o los foros híbridos de Callon et al. (2001). Los procedimientos de orden tecnocrático, que pretenden solucionar (en la versión más terminante del término) las crisis de confianza en el sistema de control alimentario mediante la "trazabilidad", $o$, en general, incrementando la "transparencia"17 , no ofrecen mecanismos para reconstruir la confianza social, perdida en gran medida como resultado de la percepción ciudadana de que las decisiones políticas se basan en el juego entre intereses empresariales, burocracias y maquinarias de partido, bastante ajustada por otra parte al estado actual de nuestras democracias. Estas soluciones requieren más confianza social en los productores de "transparencia", que terminan por hacerse democráticamente aún más opacos, sin que los mediadores y agentes visibles dejen de ser aquellos cuya actuación ha sido tantas veces cuestionada, y con tan graves y flagrantes consecuencias.

Los jurados ciudadanos y, en general, los dispositivos deliberativos, ofrecen el potencial de introducir en la conversación pública otros hilos discursivos, críticas legitimadas desde otras fuentes de representación. Siempre que el diseño ${ }^{18}$ concreto

\footnotetext{
${ }^{17}$ Véase Grossman et al. (en prensa) para una discusión de las ambigüedades en el despliegue político de este término.

${ }^{18}$ No podemos detenernos en este punto con el detenimiento que merece. El conjunto de rasgos del proceso deliberativo hace variar enormemente los resultados, la relevancia y la "validez democrática" de los mismos. Dentro de los propios jurados ciudadanos, la "versión PEALS" de los mismos (llamada significativamente DIY, o Jurados Hágalo-Usted-Mismo, como el bricolaje), tiene por ejemplo entre sus principios el que los jurados tengan la libertad de redefinir el horizonte de los problemas que tratan, lo cual configura un proceso radicalmente distinto de otros "jurados ciudadanos" más cerrados, como el que una empresa privada de comunicación organizó a instancias de la iniciativa británica GMNation.
} 
permita a los jurados reformular las claves de los problemas con sus vidas, trazar las conexiones a veces inesperadas y otras ocultas entre los riesgos, los actores y los procesos, los jurados pueden operar como constructores de públicos. Tomo aquí de John Dewey (1954[1929]) una noción algo transversal a lo que usualmente entendemos como público, y que este autor define como "aquéllos que se reconocen como afectados por las consecuencias ${ }^{19}$ de un acto privado, y que tratan de regularlo". Si ponemos el acento en esa capacidad de identificar las consecuencias de las acciones (tarea nada sencilla en condiciones de expansión tecnológica y comercial, y que hacía que el público de Dewey estuviera siempre "en búsqueda de sí mismo"), podemos imaginar a los jurados como espacios en los que actores no vinculados a las redes tecnopolíticas profesionales tendrían la oportunidad de "unir los puntos", identificar responsabilidades estructurales, y traducirlas en propuestas políticas que pasan de la "culpabilización individual" a la "responsabilidad política" (Young, 2003). Quizá precisamente por esta función, la mayor oposición (activa o por simple incomparecencia) a los foros ciudadanos se plantea desde los grupos de poder empresarial (Hendriks, 2002), que fundan al fin y a la postre su beneficio en evitar hacerse cargo de sus externalidades planetarias. Esta resistencia podría ser el mejor indicador del potencial de los dispositivos deliberativos para reconstruir democráticamente un sistema alimentario global cada vez más patológico.

\section{REFERENCIAS BIBLIOGRÁFICAS}

ACKERMAN, B. y J. FISHKIN (2002), "Deliberation day", The Journal of Political Philosophy, vol. 10, (2), pp. 119-152.

BASKIN, Y. (2002), A Plague of Rats and Rubbervines. The Growing Threat of Species Invasions, Shearwater Books, Island Press.

BENHABIB, S. (1996), "Toward a Deliberative Model of Democratic Legitimacy", en Benhabib, S. (ed), Democracy and Difference, Princeton, Princeton University Press.

BRAY, G.A., S.J. NIELSEN S y B.M. POPKIN (2004), "Consumption of high-fructose corn syrup in beverages may play a role in the epidemic of obesity", American Journal of Clinical Nutrition, vol. $79,(4)$, pp. 537-43.

CALLON, M., P. LASCOUMES y Y. BARTHE (2001), Agir dans un monde incertain. Essai sur la démocratie technique, París, Seuil.

COLINO, C. y E. DEL PINO (2003) “Un fantasma recorre Europa: renovación democrática mediante iniciativas de promoción participativa en los gobiernos locales", Ponencia presentada en las II Jornadas de Sociología Política, UNED, Madrid, 13 de diciembre.

\footnotetext{
${ }^{19}$ Este desbordamiento del marco de la acción privada es registrado por la ciencia económica como "externalidades".
} 
DAHL, R. (2003), La democracia y sus críticos, Barcelona, Paidós.

DEWEY, J. (1954[1929]), The Public and Its Problems, Athens, Swallow Press/Ohio University Press.

FIELDS, S. (2004), "The fat of the land: do agricultural subsidies foster poor health?", Environmental Health Perspectives, Vol. 112, (14), pp. 820-3.

FUNG, A. (2003), "Recipes for public spheres: Eight institutional design choices and their consequences", The Journal of Political Philosophy, Vol. 11, (2), pp. 338-367.

GROSSMAN, E., E. LUQUE y F. MUNIESA (2005), "Economies through transparency”, en GARSTEN, C. y M. LINDH DE MONTOYA (eds.), Transparency in a New Global Order: Unveiling Organizational Visions, Edward Elgar.

HABERMAS, J. (1994), Historia y crítica de la opinión pública, Barcelona, Gustavo Gili.

HENDRIKS, C. (2002), "Institutions of Deliberative Democratic Processes and Interest Groups: Roles, Tensions and Incentives", Australian Journal of Public Administration, Vol. 61, (1), pp. 64-75.

JOLY, P. B., C. MARRIS y M. A.HERMITTE (2003), "In search of 'technological democracy'. Lessons from the consensus conference on GMO's in France", Natures Sciences Societes, Vol. 11, (1), pp. 3-16.

LAKOFF, G. y M. JOHNSON (1980), Metaphors We Live By, University of Chicago Press.

LATOUR, B. (2001), "What Rules of Methods for the New Scientific Experiments?", Conferencia presentada en el 13.er Darmstadt Colloquium.

LIPPMANN, N. (1925), The Phanton Public, Nueva York, Harcourt de Brace.

LUQUE, E. (2005), "Researching environmental citizenship and its publics", Environmental Politics, (en prensa).

LUSKIN, R. C., J.S. FISHKIN y R. JOWELL (2002), "Considered opinions: Deliberative polling in Britain", British Journal of Political Science, Vol. 32, pp. 455-487.

MANIN, B. (1998), Los principios del gobierno representativo, Madrid, Alianza.

MILNER, H. (2002), Civic Literacy: How Informed Citizens Make Democracy Work, Tufts University Press, University Press of New England.

PEALS (2003). The People's Report on GM, PEALS, University of Newcastle.

PIMBERT, M.P. y T. WAKEFORD (2002), Prajateerpu: A Citizens Jury/Scenario Workshop on Food and Farming Futures for Andhra Pradesh, India, IIED, London e IDS, Sussex.

PIMBERT, M.P., T. WAKEFORD y P.V. SATHEESH (2001), "Citizens' juries on GMOs and farming futures in India", LEISA, Vol. 17, (4), pp. 27-30. 
R IS

REVISTA INTERNACIONAL DE SOCIOLOGÍA

No 40, ENERO-ABRIL, 2005

EMILIO LUQUE

PUTNAM, R. D. (2002), Solo en la bolera. Colapso y resurgimiento de la comunidad norteamericana. Barcelona, Galaxia Gutenberg/Círculo de Lectores.

SAWARD, M. (2003), “Enacting democracy”, Political Studies, Vol. 51, pp. 161-179.

YOUNG, I. (2003), "From Guilt to Solidarity: Sweatshops and Political Responsibility", Dissent, Vol. 50, (2), pp. 39-44. 